\title{
Theory of phase transformation and reorientation in single crystalline shape memory alloys
}

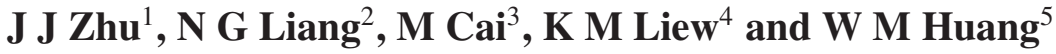 \\ ${ }^{1}$ SIMBIOS Centre, School of Computing and Creative Technologies, University of Abertay \\ Dundee, Dundee, DD1 1HG, UK \\ ${ }^{2}$ LNM Institute of Mechanics, Chinese Academy of Sciences, Beijing 100080, \\ People's Republic of China \\ ${ }^{3}$ Department of Engineering Technology, University of Houston, TX 77204, USA \\ ${ }^{4}$ Department of Building and Construction, City University of Hong Kong, \\ Kowloon, Hong Kong \\ ${ }^{5}$ School of Mechanical and Aerospace Engineering, Nanyang Technological University, \\ 639798, Singapore
}

Received 2 October 2007, in final form 28 November 2007

Published 11 January 2008

Online at stacks.iop.org/SMS/17/015041

\begin{abstract}
A constitutive model, based on an $(n+1)$-phase mixture of the Mori-Tanaka average theory, has been developed for stress-induced martensitic transformation and reorientation in single crystalline shape memory alloys. Volume fractions of different martensite lattice correspondence variants are chosen as internal variables to describe microstructural evolution. Macroscopic Gibbs free energy for the phase transformation is derived with thermodynamics principles and the ensemble average method of micro-mechanics. The critical condition and the evolution equation are proposed for both the phase transition and reorientation. This model can also simulate interior hysteresis loops during loading/unloading by switching the critical driving forces when an opposite transition takes place.
\end{abstract}

\section{Introduction}

Shape memory alloys have been recognized as promising materials for various applications for decades [1, 2]. More recently, they have also found applications as micro-actuator devices in MEMS [2-5]. The mechanism behind the unique shape memory phenomenon is the diffusionless phase transformation between the high-temperature stable phase austenite and low-temperature stable phase martensite, and reorientation among martensite variants. Modeling mechanical and microstructural behaviors of shape memory alloys is considerably complex due to the nonlinear behavior and the presence of hysteresis. Furthermore, those behaviors are temperature dependent in many cases.

In view of engineering applications, multidimensional constitutive models have been developed recently; see, for instance, [6-19]. Most previous models adopted a statistical strategy ranging from the microscale, through the mesoscale to the macroscale. However, confusion with regard to the fundamentals of micromechanical models still exists, largely due to the complexity in microstructure during the martensitic transformation. Those fundamental concepts are critical to understanding the mechanism of martensitic transformation. To name a few:

(1) What are the potential wells of martensitic transformation; habit plane variants (HPVs) or lattice correspondence variants (LCVs), or both?

(2) How can one model the interaction energy between martensite and austenite, and among martensite variants?

(3) What is the mechanism of forming an interior hysteresis loop, and how does one model it?

These three aspects of martensitic transformation are further reviewed as follows.

Lattice correspondence variant and habit plane variant. The phenomenological theory of martensite crystallography [20-23] has been successfully applied in investigating the microstructure evolution of the martensite/austenite interphase and martensite twins. This theory allows continuous 
macro-displacement with heterogeneous microscopic deformation. Hence, the Hadamard jump condition becomes an internal geometric restraint, which requires the interface between the martensite lattice correspondence variants to be a twin plane and that between austenite and martensite to be a habit plane $[24,25]$. It is noted that habit plane variants are not always the elementary units that compose the bulk martensite. Instead they are often formed by even smaller single crystalline units, which have a unique lattice correspondence with austenite [26]. In this paper, we use 'habit plane variant' to describe the morphological change of forward and reverse transformations between austenite and martensite, while employing 'lattice correspondence variant' to specify martensite lattice correspondence during the reorientation process. Since the order of symmetry of martensite is lower than that of austenite, $n$ lattice correspondence variants may be induced from one austenite crystal after martensitic phase transformation. In some shape memory alloys (e.g., $\mathrm{CuZnGa}$ and $\mathrm{CuZnAl}$, which involve $\mathrm{DO}_{3}$ austenite and 18R martensite) [27], the habit plane variant is a martensite lattice correspondence variant. In other shape memory alloy transformations, such as the $\mathrm{DO}_{3}$ austenite to $2 \mathrm{H}$ martensite transformation in CuAlNi [28-31], and B2 austenite to B19' martensite transformation in TiNi [32], the habit plane variant includes two twin-related lattice correspondence variants. Figure 1 is a sketch of a habit plane variant which consists of a pair of twinned lattice correspondence variants. Assuming $\mathbf{U}_{i}$ is the right stretch tensor of Bain strain in lattice correspondence variant $i$, the deformations in the two lattice correspondence variants $i$ and $j$ are $\mathbf{U}_{i}$ and $\mathbf{U}_{j}$, respectively. Thus, the deformation of a habit plane variant may be expressed as $\kappa \mathbf{U}_{j}+(1-\kappa) \mathbf{R} \mathbf{U}_{i}$, where $\mathbf{R}$ is the rotation of variant $i$ with regard to variant $j$. The volume fraction ratio between the twinned lattice correspondence variants $\kappa$ is a constant, as determined by the lattice parameters of austenite and martensite. In the case of $\mathrm{CuAlNi}$, the type II twin habit plane variant has one lattice correspondence variant of 0.3007 and another of $(1-0.3007)[28,29]$.

Continuum thermoelasticity, developed by Ericksen [33], Ball and James [24] and others, studies the atomic displacement and macroscopic deformation in crystalline solids undergoing a martensitic transformation. It uses the Cauchy-Born hypothesis, where minimum total free energy is required for any mechanical response. The total free energy usually comprises deformation energy (or elastic strain energy) and loading device energy (refer to equation (13) in [34], and equation (8) in [35]). The deformation energy is assumed to be a smooth function of temperature and deformation gradient. It must be frame independent and reaches its minimum at the bottom of the potential well. According to continuum thermoelasticity, each lattice correspondence variant is a potential well. The system would have $n+1$ potential wells (n lattice correspondence variants for martensite plus one for austenite). Thus, the deformation energy is minimized if the total strain equals the eigenstrain of each martensite lattice correspondence variant (without elastic strain) or zero (corresponding to austenite). It is well known that in forward transformation austenite is much easier to transform into habit plane variants than lattice correspondence variants. In order

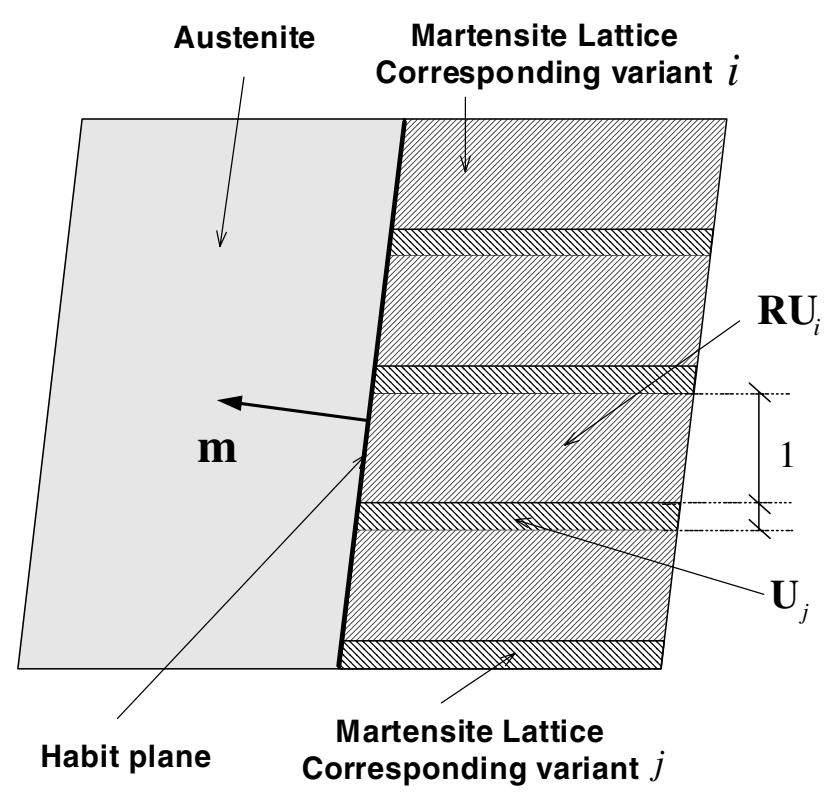

Figure 1. A habit plane variant consists of a pair of twinned lattice correspondence variants.

to obtain thermodynamic forces corresponding to habit plane variants, some previous models simply chose habit plane variants (or lattice correspondence pairs) as potential wells. It should be pointed out that a habit plane variant does not always correspond to an energy potential well. In order to establish the kinetic mechanism of martensitic transformation, the relationship between the martensite lattice correspondence variant and habit plane variant has to be identified. It is obvious that if the habit plane variants are chosen as potential wells, martensite reorientation cannot be properly described, since reorientation happens among lattice correspondence variants, which are subunits of habit plane variants. A particular example is discussed in [34]. In some of the previous models [12, 36-39], lattice correspondence variants are chosen as potential wells instead of habit plane variants. These models use a transformation 'yield' surface [40] to simulate the phase transformation process, rather than proposing thermodynamic driving forces for martensite habit plane variants. In the present paper, we demonstrate that lattice correspondence variants should be chosen as potential wells. Thermodynamic driving forces corresponding to martensite habit plane variants can still be explicitly derived out under this framework. Further, thermodynamic models for forward/reverse transformations between austenite and martensite, and reorientation among martensite variants, have been developed using this protocol.

Interaction energy. Determination of the overall deformation energy is critical in modeling the transition in shape memory alloys. Although continuum thermoelasticity has outlined the main characteristics of deformation energy (e.g. potential wells, frame independence, and smoothness of deformation gradient and temperature) and the upper bound and lower bound of deformation energy have been discussed previously [38, 41], no explicit formula for the deformation energy has been proposed. In practice, Hooke's law, linking the deformation energy with the local elastic deformation, 
applies reasonably accurately in the close vicinity of a local well [42]. We recently combined Hooke's law with the volume average scheme and suggested that the macroscopic deformation energy can be divided into two parts: the energy induced by macroscopic external stress and internal interaction energy [43]. Internal interaction energy is generated by the inhomogeneous phase transformation eigenstrain and it does not depend on the external (macroscopic) stress. Therefore, the only issue remaining is how to determine the internal interaction energy.

Previously, martensite variants have been considered as inclusions within an austenite medium and the interaction energy of a multicomponent system is estimated by Eshelby's inclusion theory. Eshelby's inclusion theory is only valid for one ellipsoidal inclusion embedded within an infinite matrix; it may not be accurate for a multi-inclusion case. Martensite/austenite interface and interface among martensite variants are planes; martensite must be in polyhedral shape rather than ellipsoid. Furthermore, inclusion theory is not applicable for the phase transformation in single crystalline shape memory alloys, in which case an austenite single crystal transforms into one martensite variant upon loading. The internal interaction energy of a multicomponent mixture can be derived by the interface operator technique [14, 44]. In Siredey's work, grains are divided into a series of domains. Each domain is assumed to end at a grain boundary and a domain meets others on one side only. By definition, only one type of martensite habit plane variant is assigned for each domain. The internal stress caused by incompatible deformation in each domain is assumed to be constant. Subsequently, a second-order polynomial function in terms of volume fractions of martensite habit plane variants can be obtained. Coefficients of this polynomial are determined by the interfacial operator. Hence, they are dependent upon the microstructure of a particular shape memory alloy, and can be determined at a microscopic level.

Mori and Tanaka [45] have proven that if two phases are mixed together in a random and uniform fashion, the interaction energy produced by the incompatible deformation is proportional to $z(1-z)$ [46]. Here $z$ is the volume fraction of one phase. Similar formulae have been used for shape memory alloys [47-51]. Unfortunately, this simple closed-form solution is only for two-phase mixtures. In shape memory alloys, there are $n$ martensite variants plus one for austenite. Thus, the interaction energy in an $(n+1)$ component mixture has to be considered. We recently extended the Mori-Tanaka theory to an $(n+1)$-component mixture [43]. It can subsequently be concluded that the inclusion theory is only applicable for one ellipsoidal inclusion case, while Mori-Tanaka theory is valid for the multi-inclusion case. The volume fraction of martensite has to be much less then 1 in the inclusion theory, while it can be any value between 0 and 1 in Mori-Tanaka theory. It is noted that the interaction energy of a two-component mixture estimated by the inclusion theory also appears as a second-order polynomial. But this is quite different from that predicted by Mori-Tanaka theory. The coefficient is determined by an Eshelby tensor in the former, while the calibration can be done by a macroscale experiment

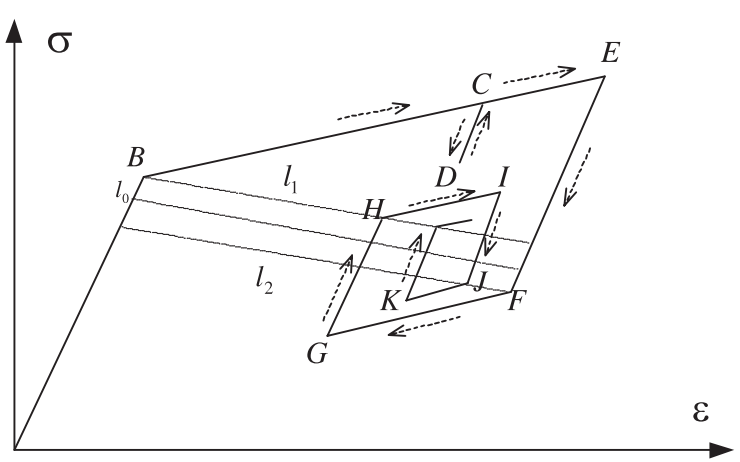

Figure 2. Sketch of an interior hysteresis loop.

in the latter. In addition, it has been proven that the total number of independent constants in the interaction energy is no more than the number of martensite lattice correspondence variants [43].

Interior hysteresis loop. A complex interior hysteresis loop was observed in uniaxial tensile tests in single crystalline shape memory alloys [47-53], and a similar phenomenon has been reported in martensite reorientation between two martensite lattice correspondence variants [34]. These interior hysteresis loops were given various names, such as internal elasticity, internal yield and internal recovery in the literature (for example figure 2 in [48], figures 1 and 4 in [49], figures 4, 5 and 8 in [50]). Considering an isothermal loading/unloading process as shown in figure 2, phase transformation starts at $\mathrm{B}$ upon loading. If unloading at $\mathrm{C}$, it moves from $\mathrm{C}$ to $\mathrm{D}$. During reloading from $\mathrm{D}$, it returns to $\mathrm{C}$ elastically. As there is no reverse phase transformation upon unloading, the last transformation state in the forward transition at point $\mathrm{C}$ is remembered. The forward transition will not restart until reloading to $\mathrm{C}$. If unloading from point $\mathrm{E}$, a reverse transition appears $(\mathrm{E} \rightarrow \mathrm{F} \rightarrow \mathrm{G}$ as shown in figure 2); the last transformation state in the forward transition at point $\mathrm{E}$ is obliterated. During reloading from $\mathrm{G}$, the forward transformation restarts at $\mathrm{H}$. A similar phenomenon happens in the reverse transformation and reorientation (e.g., tension/compression cycling). Oblivion plays an important role in the formation of an interior hysteresis loop, which happens when an internal state crosses lines $l_{1}$ and $l_{2}$ in figure 2 . Huo and Müller [50] concluded that the interior hysteresis loop is due to history-dependent pseudoelasticity, and the fraction of the last transformation state is remembered. Fedelich and Zanzotto [48] suggested choosing the fraction of martensite variant in the last transformation as an additional internal variable to describe these loops. The present paper only deals with transformations with a stable hysteresis loop; refer to $[54,55]$ for the instability of the phase transformation.

We aim at developing a robust micro-mechanical model to address the above three issues in this paper. In section 2 , the macroscopic Gibbs free energy is expressed as a function of volume fractions of different martensite lattice correspondence variants. Basic transition processes (e.g. forward/reverse transformations and reorientation) and the transition law, and the prediction of an interior hysteresis loop are investigated in section 3. Conclusions and outlook of the proposed 


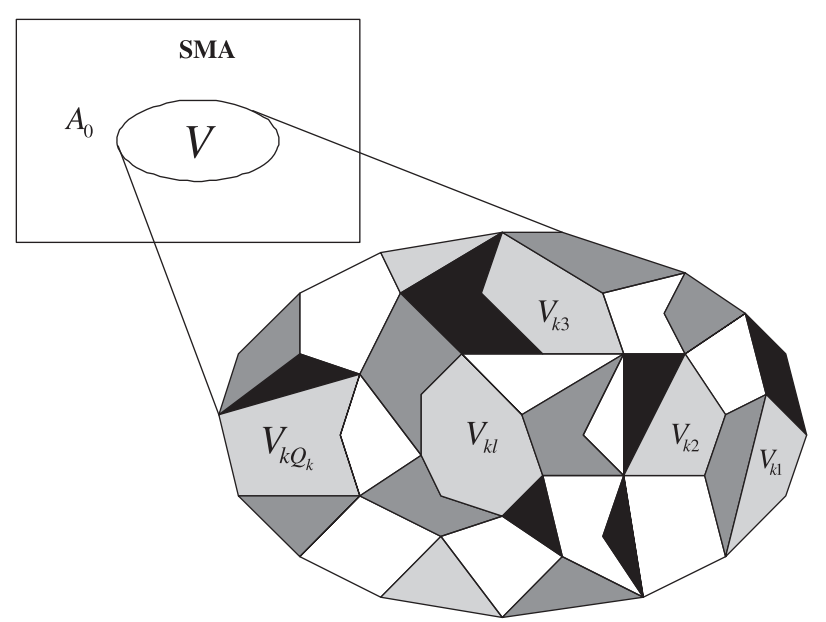

Figure 3. Sketch of a representative volume element. Areas with different shades stand for different martensite lattice correspondence variants. $k=0,1,2 \ldots n, l=1,2, \ldots Q_{k}$.

thermodynamic models for shape memory alloys are given in section 4.

\section{Thermodynamics of martensitic transformation}

In this section, the non-equilibrium thermodynamics of martensitic transformation is investigated using the ensemble average method. Martensite lattice correspondence variants are chosen as internal variables to describe the microstructural configuration. Potential differences between the martensite lattice correspondence variant and austenite are derived from macroscopic Gibbs free energy.

A single crystalline shape memory alloy is pure austenite if the material is stress-free and the temperature is higher than its austenite finish temperature. Taking a representative volume element (with volume $V$ and its boundary $A_{0}$ ) from a single crystal, a martensitic transformation may be induced by either stressing or cooling (figure 3 ). In both cases, the microstructural evolution is by means of the growth of martensite at the expense of austenite until, ideally, no more austenite exists.

Within martensite, there are $n$ possible lattice correspondence variants. Therefore, in a representative volume element, $n+1$ components may coexist (let $k=1,2 \ldots n$ represent $n$ martensite lattice correspondence variants, and $k=0$ for austenite). As shown in figure 3, one martensite lattice correspondence variant may be found inside many different regions in the representative volume element. The same martensite lattice correspondence variants are represented by the same shade in figure 3 . We assume that the number of regions with a particular type of martensite lattice correspondence variant $k$ is $Q_{k}$ and the volume of the $l$ th $\left(l=1,2 \ldots Q_{k}\right)$ region with martensite lattice correspondence variant of type $k$ is $V_{k l}$ (with boundary $A_{k l}$ ). For consistency, all vectors and tensors are in bold face and the summation convention for repeated indices is not used in this paper. For simplicity, we only consider elastic deformation and deformation due to the phase transformation; and we assume that the two phases (martensite and austenite) share the same thermal and physical properties. Thus the following assumptions are made:

(1) The total deformation can be divided into two parts: namely, the elastic deformation and deformation induced by the transformation.

(2) The elastic modulus ( $\mathbf{L})$ of austenite is the same as that of martensite. The elastic compliance tensor is given by $\mathbf{M}=\mathbf{L}^{-1}$

(3) The specific heat $C_{V}$ and heat conduction coefficient $k_{0}$ of austenite and martensite are the same.

(4) Martensite in three-dimensional space is randomly and uniformly distributed.

(5) The density $\rho$, temperature field $T$, heat flow $\mathbf{q}$ and specific heat source $\dot{r}$ are uniform in the representative volume element. Only macroscopic heat conduction is considered and the heat conduction among different phases within the representative volume element is ignored.

(6) Thermal expansion is ignored.

According to assumption 1, the total strain $\mathbf{e}_{k l}$ is the sum of elastic strain and phase transition strain:

$$
\mathbf{e}_{k l}=\mathbf{e}_{k l}^{\mathrm{e}}+\mathbf{E}_{k}^{\mathrm{tr}} \quad\left(l=1,2, \ldots, Q_{k}\right)
$$

where $\mathbf{e}_{k l}^{\mathrm{e}}$ is the elastic strain and $\mathbf{E}_{k}^{\mathrm{tr}}$ is the eigenstrain due to the phase transformation from austenite to type $k$ martensite lattice correspondence variant. It is noticed that, for $V_{k l}\left(l=1,2, \ldots, Q_{k}\right)$, which corresponds to the same variant of type $k$ located at various places, their phase transformation eigenstrains are the same although their elastic strain $\mathbf{e}_{k l}^{\mathrm{e}}$ may be different.

If the point group of austenite is given by $\mathbb{P}_{\mathrm{a}}$ and that of martensite is $\mathbb{P}_{m}$, then $\mathbb{P}_{m}$ is a subgroup of $\mathbb{P}_{a}$ [43]. Let $\mathbb{L}$ be the left coset of $\mathbb{P}_{m}$ in $\mathbb{P}_{a}$, i.e.,

$$
\mathbb{P}_{\mathrm{a}}=\mathbb{L} \otimes \mathbb{P}_{\mathrm{m}}
$$

We have

$$
\mathbf{E}_{k}^{\mathrm{tr}}=\mathbf{R}_{k} \mathbf{E}_{1}^{\mathrm{tr}} \mathbf{R}_{k}^{T}, \quad \mathbf{R}_{k} \in \mathbb{L},
$$

where $k=1,2,3, \ldots n$, and $n$ is the total number of lattice correspondence variants. For austenite $(k=0)$, there is no phase transformation term but elastic deformation only, i.e.,

$$
\mathbf{e}_{0 l}=\mathbf{e}_{0 l}^{\mathrm{e}} \quad\left(l=1,2, \ldots, Q_{0}\right) .
$$

Assuming that the specific internal energies and specific entropies of austenite and martensite at the reference temperature $T_{0}$ are $u_{0}^{\mathrm{A}}, u_{0}^{\mathrm{M}}, h_{0}^{\mathrm{A}}$, and $h_{0}^{\mathrm{M}}$, respectively, then the specific entropy $\eta^{\mathrm{M}}$, elastic energy $\phi_{k l}$, and specific internal energy $u_{k l}$ for martensite $V_{k l}(k \neq 0)$ may be expressed as

$$
\begin{gathered}
\rho \eta^{\mathrm{M}}(T)=h_{0}^{\mathrm{M}}+C_{V} \ln \left(\frac{T}{T_{0}}\right) \quad(k \neq 0), \\
\phi_{k l}=\frac{1}{2}\left(\mathbf{e}_{k l}-\mathbf{E}_{k}^{\mathrm{tr}}\right): \mathbf{L}:\left(\mathbf{e}_{k l}-\mathbf{E}_{k}^{\mathrm{tr}}\right)=\frac{1}{2} \boldsymbol{\sigma}_{k l}: \mathbf{M}: \boldsymbol{\sigma}_{k l}, \\
\rho u_{k l}=u_{0}^{\mathrm{M}}+C_{V}\left(T-T_{0}\right)+\phi_{k l} \quad(k \neq 0),
\end{gathered}
$$

where $l=1,2, \ldots, Q_{k}$ and $\sigma_{k l}$ is the stress applied on the martensite volume specified by $k l$. For austenite $V_{0 l}(k=0)$, 
the specific entropy $\eta^{\mathrm{A}}$, elastic energy $\phi_{0 l}$, and specific internal energy $u_{0 l}$ are

$$
\begin{gathered}
\rho \eta^{\mathrm{A}}(T)=h_{0}^{\mathrm{A}}+C_{V} \ln \left(\frac{T}{T_{0}}\right) \quad(k=0), \\
\phi_{0 l}=\frac{1}{2} \mathbf{e}_{0 l}: \mathbf{L}: \mathbf{e}_{0 l}=\frac{1}{2} \boldsymbol{\sigma}_{0 l}: \mathbf{M}: \boldsymbol{\sigma}_{0 l}, \\
\rho u_{0 l}=u_{0}^{\mathrm{A}}+C_{V}\left(T-T_{0}\right)+\phi_{0 l},
\end{gathered}
$$

where $l=1,2, \ldots, Q_{0}$. Hence, $\mathbf{e}_{k l}=\mathbf{E}_{k}^{\mathrm{tr}}(k=1,2, \ldots n)$ and $\mathbf{e}_{0 l}=0$ form $n+1$ potential wells.

For any given microscopic quantity $\psi$, define its corresponding macroscopic average as

$$
\langle\psi\rangle=\frac{1}{\rho V} \iiint_{V} \rho \psi \mathrm{d} V=\frac{1}{\rho V} \sum_{k=0}^{n} \sum_{l=1}^{Q_{k}} \iiint_{V_{k l}} \rho \psi_{k l} \mathrm{~d} V,
$$

where $\rho$ is the macroscopic density of the representative volume element. Recalling assumption (4), one has

$$
\langle\psi\rangle=\frac{1}{V} \iiint_{V} \psi \mathrm{d} V=\frac{1}{V} \sum_{k=0}^{n} \sum_{l=1}^{Q_{k}} \iiint_{V_{k l}} \psi_{k l} \mathrm{~d} V .
$$

Let the volume fraction of type $k$ lattice correspondence variant in a representative volume element be

$$
z_{k}=\frac{V_{k}}{V}
$$

where

$$
V_{k}=\sum_{l=1}^{Q_{k}} V_{k l}
$$

Obviously,

$$
\sum_{k=0}^{n} z_{k} \equiv 1
$$

The macroscopic volume average strain (i.e. in equation (11) $\psi=\mathbf{e})$ may be defined by $[56,57]$

$$
\mathbf{E}=\langle\mathbf{e}\rangle=\frac{1}{V} \sum_{k=0}^{n} \sum_{l=1}^{Q_{k}} \iiint_{V_{k l}} \mathbf{e}_{k l} \mathrm{~d} V
$$

where $\mathbf{e}$ is the microscopic strain; thus

$$
\mathbf{E}=\mathbf{E}^{\mathrm{e}}+\mathbf{E}^{\mathrm{tr}}
$$

where $\mathbf{E}^{\mathrm{e}}$ and $\mathbf{E}^{\mathrm{tr}}$ are the macroscopic elastic strain and macroscopic phase transformation strain, respectively, and

$$
\begin{gathered}
\mathbf{E}^{\mathrm{e}}=\left\langle\mathbf{e}^{\mathrm{e}}\right\rangle=\frac{1}{V} \sum_{k=0}^{n} \sum_{l=1}^{Q_{k}} \iiint_{V_{k l}} \mathbf{e}_{k l}^{\mathrm{e}} \mathrm{d} V, \\
\mathbf{E}^{\mathrm{tr}}=\sum_{k=1}^{n} z_{k} \mathbf{E}_{k}^{\mathrm{tr}}
\end{gathered}
$$

where $\mathbf{e}^{\mathrm{e}}$ is the microscopic elastic strain. Similarly, the macroscopic average of specific entropy $\xi$, elastic energy $\Phi$ and specific internal energy $U$ can be defined as

$$
\xi=\langle\eta\rangle
$$

$$
\begin{aligned}
& \Phi=\langle\phi\rangle, \\
& U=\langle u\rangle .
\end{aligned}
$$

Substituting equations (5)-(10) into (20)-(22), and referring to equation (21), one has

$$
\begin{aligned}
\rho \xi & =\langle\rho \eta\rangle=z_{0}\left[h_{0}^{\mathrm{A}}+C_{V} \ln \left(\frac{T}{T_{0}}\right)\right] \\
& +\sum_{k=1}^{n} z_{k}\left[h_{0}^{\mathrm{M}}+C_{V} \ln \left(\frac{T}{T_{0}}\right)\right], \\
\rho U & =\langle\rho u\rangle=z_{0}\left[u_{0}^{\mathrm{A}}+C_{V}\left(T-T_{0}\right)\right] \\
& +\sum_{k=1}^{n} z_{k}\left[u_{0}^{\mathrm{M}}+C_{V}\left(T-T_{0}\right)\right]+\Phi .
\end{aligned}
$$

Supposing that the macroscopic stress applied on volume $V$ is $\boldsymbol{\Sigma}$, it is the conjugate stress with macroscopic strain $\mathbf{E}$ [56-59], which has to satisfy

$$
\frac{1}{V} \iiint_{V} \sigma: \dot{\mathbf{e}} \mathrm{d} V=\boldsymbol{\Sigma}: \dot{\mathbf{E}} .
$$

It would be convenient to define all stress and strain tensors in a reference configuration, say the $\sigma$ (or $\boldsymbol{\Sigma}$ ) second PiolaKirchhoff stress tensor (or Lagrangian Piola-Kirchhoff stress) and the e (or E) Lagrangian strain tensor. According to [43], the total macroscopic deformation energy is a sum of macroelastic energy in the absence of transformation and the internal interaction energy due to martensitic transformation in the absence of external stress, that is

$$
\Phi=\frac{1}{2} \boldsymbol{\Sigma}: \mathbf{M}: \boldsymbol{\Sigma}+f^{\mathrm{s}},
$$

where $f^{\mathrm{s}}$ is the 'stored elastic energy'-internal energy due to the internal stress field $\sigma_{\text {int }}$ (induced by eigenstrain $\varepsilon^{*}$ ).

$$
f^{\mathrm{s}}=\Phi_{\text {int }}=-\frac{1}{2 V} \iiint_{V} \boldsymbol{\sigma}_{\text {int }}: \boldsymbol{\varepsilon}^{*} \mathrm{~d} V
$$

and

$$
\boldsymbol{\varepsilon}^{*}= \begin{cases}\mathbf{E}_{k}^{\mathrm{tr}} & \text { if } \mathbf{x} \in V_{k l} \\ 0 & \text { if } \mathbf{x} \in V-\boldsymbol{\Omega},\end{cases}
$$

is the microscopic eigenstrain of the phase transformation; $\Omega$ denotes the total volume occupied by martensite.

It has been proved that $f^{\text {s }}$ does not directly depend upon the external stress field; it is a second-order polynomial function of martensite lattice correspondence variant fractions [43], i.e.,

$$
f^{\mathrm{s}}=\Phi_{\text {int }}=A z_{0}\left(1-z_{0}\right)+\frac{1}{2} \sum_{k=1}^{n} \sum_{\substack{l=1 \\ l \neq k}}^{n} B_{k l} z_{k} z_{l},
$$

where $A$ and $B_{k l}$ are material constants describing the interaction between austenite and martensite and among martensite variants, respectively. $A$ and $B_{k l}$ should be calibrated experimentally at macroscopic scales. It was also proved that the maximum total number of independent $B_{k l}$ is $n-1$ [43]. Substituting equation (26) into (24) results in

$\rho U=\frac{1}{2} \boldsymbol{\Sigma}: \mathbf{M}: \boldsymbol{\Sigma}+z_{0}\left[u_{0}^{\mathrm{A}}+C_{V}\left(T-T_{0}\right)\right]$

$$
+\sum_{k=1}^{n} z_{k}\left[u_{0}^{\mathrm{M}}+C_{V}\left(T-T_{0}\right)\right]+f^{\mathrm{s}} \text {. }
$$


The total work includes work done by the surface force and the body force, respectively. We define the total work rate per unit volume as

$$
\dot{W}=\frac{1}{V}\left[\iint_{A_{0}} \boldsymbol{\sigma}:(\mathbf{v} \otimes \mathbf{n}) \mathrm{d} A+\iiint_{V} \mathbf{f} \cdot \mathbf{v} \mathrm{d} V\right],
$$

where $\mathbf{v}$ is the velocity, $\mathbf{n}$ is the outwards normal direction of boundary $A_{0}$, and $\mathbf{f}$ is the body force. Note the equilibrium of an object subjected to an external stress and body force:

$$
\frac{\partial \boldsymbol{\sigma}}{\partial \mathbf{x}}+\mathbf{f}=0
$$

Referring to equation (25), equation (31) can be rewritten as

$$
\dot{W}=\frac{1}{V} \iiint_{V} \sigma: \dot{\mathbf{e}} \mathrm{d} V=\Sigma: \dot{\mathbf{E}} .
$$

One may choose $\left(\Sigma, z_{k}, T\right)$ as independent variables at the macroscopic scale. The macroscopic Gibbs free energy function is defined by

$$
\rho G=\rho U-T \rho \xi-\Sigma: \mathbf{E} .
$$

By substituting equations (17), (19), (23), and (30) into (34), we obtain that

$$
\begin{aligned}
\rho G & =\rho U-T \rho \xi-\Sigma:\left(\mathbf{E}^{\mathrm{e}}+\sum_{k=0}^{n} z_{k} \mathbf{E}_{k}^{\mathrm{tr}}\right) \\
= & \rho U-T \rho \xi-\Sigma: \mathbf{M}: \Sigma-\Sigma:\left(\sum_{k=0}^{n} z_{k} \mathbf{E}_{k}^{\mathrm{tr}}\right) \\
= & z_{0}\left[u_{0}^{\mathrm{A}}+C_{V}\left(T-T_{0}\right)\right]+\sum_{k=1}^{n} z_{k}\left[u_{0}^{\mathrm{M}}+C_{V}\left(T-T_{0}\right)\right] \\
& +f^{\mathrm{s}}-T\left\{z_{0}\left[h_{0}^{\mathrm{A}}+C_{V} \ln \left(\frac{T}{T_{0}}\right)\right]\right. \\
& \left.+\sum_{k=1}^{n} z_{k}\left[h_{0}^{\mathrm{M}}+C_{V} \ln \left(\frac{T}{T_{0}}\right)\right]\right\} \\
& -\Sigma:\left(\sum_{k=0}^{n} z_{k} \mathbf{E}_{k}^{\mathrm{tr}}\right)-\frac{1}{2} \Sigma: \mathbf{M}: \Sigma .
\end{aligned}
$$

From equation (35), we get

$$
\mathbf{E}=-\rho \frac{\partial G}{\partial \Sigma}=\mathbf{M}: \Sigma+\sum_{k=0}^{n} z_{k} \mathbf{E}_{k}^{\mathrm{tr}}
$$

and

$$
\xi=-\frac{\partial G}{\partial T}
$$

Let $\Xi_{k}$ be potential difference between type $k$ martensite lattice correspondence variant and austenite, then

$$
\Xi_{k}=-\rho \frac{\partial G}{\partial z_{k}}=\Sigma: \mathbf{E}_{k}^{\mathrm{tr}}+(\Delta h T-\Delta u)-\frac{\partial f^{\mathrm{s}}}{\partial z_{k}},
$$

where

$$
\begin{gathered}
\Delta h=h_{0}^{\mathrm{M}}-h_{0}^{\mathrm{A}} \\
\Delta u=u_{0}^{\mathrm{M}}-u_{0}^{\mathrm{A}} .
\end{gathered}
$$

The phase equilibrium temperature is determined by $T^{\mathrm{eq}}=$ $\frac{\Delta u}{\Delta h}$.

Letting $\rho T \eta^{*}$ be the total entropy product in representative volume element $(V)$, which includes contributions from phase transformation and heat conduct, the second thermodynamic principle may be written as

$$
\rho T \eta^{*}=\sum_{k=1}^{n} \Xi_{k} \dot{z}_{k}-\frac{1}{T} \mathbf{q} \cdot \nabla T \geqslant 0 .
$$

\section{Transition process}

In this section, we use progress variables and stoichiometric coefficients to describe the transition process in shape memory alloys. The thermodynamic driving force for an individual progress variable is obtained. Critical conditions and evolution equations for the forward transformation, reverse transformation and reorientation are derived.

\subsection{Basic transition process}

In section 2, we have defined and derived potentials for each martensite lattice correspondence variant and austenite. However, austenite and martensite lattice correspondence variants may simultaneously vary their volume fractions in a real transition process. For instance, upon cooling in a piece of stress-free austenite shape memory alloy, all martensite habit plane variants may be produced equally and grow simultaneously at a critical temperature (also called selfaccommodation martensite). For a simplest transition process, the phase transformation starts with austenite transforming into a habit plane variant, which may include two lattice correspondence variants in some shape memory alloys. On the other hand, reorientation is a transition process between two martensite lattice correspondence variants.

In a given transition process (denoted by subscript $l$ ), the volume fractions of austenite and each martensite lattice correspondence variant vary proportionally. The rate among these variations may be described in terms of stoichiometric coefficients $\left(v_{0 l}, v_{1 l}, v_{2 l}, \ldots, v_{n l}\right)$. Mass conservation yields

$$
\sum_{k=0}^{n} v_{k l}=0
$$

The progress state of the process may be presented by a progress variable $\zeta_{l}$. It varies between 0 and 1 , where $\zeta_{l}=0$ is for the beginning of a process and $\zeta_{l}=1$ when the process finishes. Thus, the variations of volume fractions of austenite and each martensite lattice correspondence variant due to this transition process can be expressed by

$$
\dot{z}_{k}=v_{k l} \dot{\zeta}_{l} \quad(k=0,1,2 \ldots n)
$$

From equation (42), the total potential variation rate of martensite takes the form of

$$
\sum_{k=1}^{n} \Xi_{k} \dot{z}_{k}=\left[\sum_{k=1}^{n} \Xi_{k} v_{k l}\right] \dot{\zeta}_{l}=\Pi_{l} \dot{\zeta}_{l},
$$


where

$$
\Pi_{l}=\sum_{k=1}^{n} \Xi_{k} v_{k l} .
$$

This is the thermodynamic driving force for a specific progress variable $\zeta_{l}$. Equation (44) shows that the thermodynamic driving force of any transition process is a linear combination of thermodynamic driving forces of lattice correspondence variants. The combination coefficient is the stoichiometric coefficient of the transition process.

Any complex transition process can be considered as a combination of these basic processes, namely, forward transformation, reverse transformation and reorientation. In the case where the habit plane variant is the lattice correspondence variant, the stoichiometric coefficient of the forward phase transformation (from austenite to type $l$ martensite lattice correspondence variant) is

$$
\begin{aligned}
& \text { element } 0 \text { element } l \\
& \text { 企业 } \\
& \left(v_{0 l}, v_{1 l}, v_{2 l}, \ldots, v_{n l}\right)=(-1,0, \ldots 0,1,0, \ldots 0) .
\end{aligned}
$$

The first element in the right-hand side of equation (45) is -1 , the $l$ th element is +1 , and all other columns are 0 . Substitution of equation (45) into (44) yields

$$
\Pi_{l}=\Xi_{l} .
$$

If the habit plane variant is not a subunit of martensite, it has to be composed of a pair of twinned martensite lattice correspondence variants. Under this condition, in a forward phase transformation austenite transforms into two twinned martensite lattice correspondences (e.g. element $j$ and $i$ ). Then, the stoichiometric coefficient of this process is written as

$$
\begin{aligned}
& \text { element } 0 \quad \text { element } i \quad \text { element } j \\
& \text { 企企业 } \\
& \left(v_{0 l}, v_{1 l}, v_{2 l}, \ldots v_{n l}\right)=(-1,0, \ldots 0,1-\kappa, 0, \ldots 0, \kappa, 0, \ldots 0) \text {. }
\end{aligned}
$$

Here, $\kappa$ is the volume fraction ratio of two twinned martensite lattice correspondence variants. On substitution of equation (47) into (44) and considering equation (38), we have

$$
\begin{aligned}
& \Pi_{l}=(1-\kappa) \Xi_{i}+\kappa \Xi_{j} \\
& \quad=\Sigma: \mathbf{E}_{l}^{*}+(\Delta h T-\Delta u)-\left[(1-\kappa) \frac{\partial f^{\mathrm{s}}}{\partial z_{i}}+\kappa \frac{\partial f^{\mathrm{s}}}{\partial z_{j}}\right],
\end{aligned}
$$

where

$$
\mathbf{E}_{l}^{*}=(1-\kappa) \mathbf{E}_{i}^{\mathrm{tr}}+\kappa \mathbf{E}_{j}^{\mathrm{tr}}
$$

is the phase transformation eigenstrain of the habit plane variant. Equations (48) and (49) give the explicit expressions of the thermodynamic driving force of habit plane variants in terms of the phase transformation eigenstrain of habit plane variants. Although equation (48) is similar to previous models developed based on choosing martensite habit plane variants as potential wells (e.g. [7, 9, 11, 14-16, 18, 19, 60]), it is important to point out that martensite habit plane variants are not the potential wells as observed in some shape memory alloys, where habit plane variants consist of twined martensite correspondence variant pairs [29-31]. This similarity also indicates that our model is robust in supporting the previous models from a more fundamental sense.

We denote the volume fraction of a habit plane variant as $z_{l}^{*}$ to distinguish it from the lattice correspondence variant, as traditionally $z_{l}^{*}$ was denoted as $z$. In the forward phase transformation process, $z_{l}^{*}$ can also be considered as a progress variable, i.e. $\zeta_{l}=z_{l}^{*}\left(\zeta_{l}=0\right.$ stands for the case of whole material being austenite, while $\zeta_{l}=1$ stands for the case of whole material being type $l$ martensite habit plane variant).

The stoichiometric coefficient in reorientation (from lattice correspondence variant $i$ to lattice correspondence variant $j$ ) can be presented as

$$
\begin{aligned}
& \text { element } i \quad \text { element } j \\
& \text { 企业 } \\
& \left(v_{0 l}, v_{1 l}, v_{2 l} \cdots v_{n l}\right)=(0, \ldots, 0,-1,0, \ldots, 0,1,0, \ldots, 0) \text {. }
\end{aligned}
$$

Substituting equation (50) into (44) and using equation (38) gives [61]

$$
\begin{aligned}
\Pi_{l}= & \Xi_{j}-\Xi_{i}=\Sigma:\left(\mathbf{E}_{j}^{\mathrm{tr}}-\mathbf{E}_{i}^{\mathrm{tr}}\right)-\left(\frac{\partial f^{\mathrm{s}}}{\partial z_{j}}-\frac{\partial f^{\mathrm{s}}}{\partial z_{i}}\right), \\
& (i, j=1, \ldots n, i \neq j) .
\end{aligned}
$$

In this transition process, the volume fraction of martensite lattice correspondence variant $j$ may also be taken as a progress variable. $\zeta_{l}=0$ stands for the situation where the whole material is lattice correspondence variant $i$, and $\zeta_{l}=1$ stands for the case of the whole material being lattice correspondence variant $j$. It can also be seen from equation (51) that the reorientation between the two adjacent martensite lattice correspondence variants is independent of temperature $T$.

According to equation (44), if the stoichiometric coefficients of two basic processes $\zeta_{l}$ and $\zeta_{\mathrm{m}}$ satisfy $\nu_{k l}=$ $-v_{k m}$, then $\Pi_{l}=-\Pi_{\mathrm{m}}$. Therefore, $\zeta_{\mathrm{m}}$ can also be represented as $-\zeta_{l}$, or vice versa. It is apparent that $\zeta_{l}$ and $\zeta_{\mathrm{m}}$ are mutual reverse processes of each other. For the process variable $\zeta_{l}, \dot{\zeta}_{l}>0$ is the forward process, while $\dot{\zeta}_{l}<0$ is the reverse process. Similarly, $\dot{z}_{l}^{*}>0$ stands for the forward transformation process, and $\dot{z}_{l}^{*}<0$ for reverse transformation process. Reorientation from the lattice correspondence variant $j$ to the lattice correspondence variant $i$ may be considered as a reverse process of the reorientation from the lattice correspondence variant $i$ into the lattice correspondence variant $j$.

Supposing the number of martensite habit plane variants is $H$, there will be a total of $2 H$ processes (i.e. $H$ forward transformation processes and $H$ reverse transformation processes). Because there are $n(n-1)$ reorientation processes among $n$ martensite lattice correspondence variants, the total number of basic transformation process is given by

$$
M_{0}=2 H+n(n-1) \text {. }
$$

Otherwise the total number of basic transformation process will be $H+\frac{n(n-1)}{2}$ if two mutual reverse processes are considered as one independent process. In the present paper we take two mutual reverse processes as two processes, so that 
for each basic process we always have $\dot{\zeta}_{l} \geqslant 0$. Any complex transformation process can be considered as a combination of basic transition processes. The variation of volume fractions of martensite lattice correspondence variants can be expressed in terms of the variation of $M_{0}$ basic progress variables $\dot{\zeta}_{l}(l=$ $\left.1,2, \ldots M_{0}\right)$,

$$
\dot{z}_{k}=\sum_{l=1}^{M_{0}} v_{k l} \dot{\zeta}_{l} \quad(k=0,1,2 \ldots n)
$$

For a given process, not all basic processes are involved (or in action). If basic process $l$ is inactive, its variation is 0 , i.e., $\dot{\zeta}_{l}=0$.

After substituting equation (53) into (40) and considering equation (44), the second law of thermodynamics can be expressed as [61]

$$
\rho T \eta^{*}=\sum_{l=1}^{M_{0}} \Pi_{l} \dot{\zeta}_{l}-\frac{1}{T} \mathbf{q} \cdot \nabla T \geqslant 0 .
$$

The strong form of equation (54) may be given as

$$
-\frac{1}{T} \mathbf{q} \cdot \nabla T \geqslant 0
$$

and

$$
\Pi_{l} \dot{\zeta}_{l} \geqslant 0
$$

\subsection{Transition law}

Transition laws of these $M_{0}$ basic transformation processes are considered in this section. According to the experimental results in [50], the critical condition for starting a phase transformation is

$$
\Pi_{l}=\Pi_{l}^{\mathrm{c} \pm}, \quad(l=1,2, \ldots H),
$$

where ' + ' stands for the forward transformation, and '-' for the reverse transformation. $\Pi_{l}^{\mathrm{c}+}>0$ and $\Pi_{l}^{\mathrm{c}-}<0$ correspond to the critical thermodynamic driving forces for the start of the forward transformation and the reverse transformation, respectively.

The variation of volume fraction of a martensite habit plane variant is proportional to the variation of its thermodynamic driving force during a forward or reverse phase transformation $[49,50]$. Thus, the phase transformation evolution equation can be expressed as

$$
\begin{aligned}
& 2\left(\lambda+\mu \frac{1}{z_{0}}\right) \dot{z}_{l}^{*}=\dot{\Pi}_{l}^{\mathrm{c}+}=\dot{\Pi}_{l} \quad \text { (forward transformation) } \\
& 2\left(\lambda+\mu \frac{1}{z_{l}^{*}}\right) \dot{z}_{l}^{*}=\dot{\Pi}_{l}^{\mathrm{c}-}=\dot{\Pi}_{l} \quad \text { (reverse transformation). }
\end{aligned}
$$

Here, $\lambda$ and $\mu$ are non-negative material constants. $\lambda$ is employed to illustrate the linear hardening phenomenon during the transformation process (i.e. the slope of lines BE, HI and FG in figure 2 is $2 \lambda$ ); $\mu$ is introduced to describe the nonlinear hardening behavior when the transformation approaches its end (i.e. $z_{0} \rightarrow 0$ or $z_{l}^{*} \rightarrow 0$ ). In the forward transformation, $\Pi_{l}^{\mathrm{c}+}$ increases with the thermodynamic driving force $\Pi_{l}$; while in the reverse transformation, $\Pi_{l}^{c-}$ decreases with the decrease of thermodynamic driving force $\Pi_{l}$. Once nucleation starts in the forward transformation, $\Pi_{l}^{\mathrm{c}-}$ moves back to the maximum value $\left(\Pi_{0}^{-}\right)$instantly. On the other hand, when the reverse transformation starts, $\Pi_{l}^{\mathrm{c}+}$ returns to the minimum value $\left(\Pi_{0}^{+}\right)$. $\Pi_{0}^{+}$and $\Pi_{0}^{-}$are materials constant with $\Pi_{0}^{+}>0$ and $\Pi_{0}^{-}<0$. In most cases, $\Pi_{0}^{+}=-\Pi_{0}^{-}=\Pi_{0}$.

Shown in figure 2 , lines $l_{1}, l_{0}$ and $l_{2}$ correspond to the cases where $\Pi=\Pi_{0}, \Pi=0$ and $\Pi=-\Pi_{0}$, respectively. The size of the hysteresis loop depends not only on the distance between $l_{1}$ and $l_{2}$ (i.e., the magnitude of $\Pi_{0}$ ), but also on the slope of $l_{0}$ (i.e., $2 A$, as also defined by equation (29)). The parameter $\Pi_{0}$ stands for the internal friction in the phase transformation, while $A$ represents the elastic energy stored in the interphase between martensite and austenite. Lexcellent [19] and others neglected the stored energy and took $A=0$. Thus, the internal friction is the only cause of hysteresis in phase transformation. On the other hand, Huo and Müller [50] considered $\Pi_{0}=0$, i.e., the hysteresis is due to the interphase elastic energy only. In such a case, the lines $l_{1}, l_{0}$ and $l_{2}$ in figure 2 coincide. It is also worth mentioning that for a material with apparent hardening behavior, the following relation holds (see also figure 2 and compare the slopes of line $\mathrm{BE}$ and $l_{0}$ ):

$$
\lambda>A \text {. }
$$

Referring to equation (58), in the forward transformation $\left(\dot{z}_{l}^{*}>\right.$ $0)$, one has

$$
\Pi_{l} \dot{z}_{l}^{*}=\frac{z_{0}}{2\left(\lambda z_{0}+\mu\right)} \Pi_{l} \dot{\Pi}_{l}=\frac{z_{0}}{4\left(\lambda z_{0}+\mu\right)} \frac{\mathrm{d}}{\mathrm{d} t}\left(\Pi_{l}^{\mathrm{c}+}\right)^{2} \geqslant 0 .
$$

In the reverse transformation $\left(\dot{z}_{l}^{*}<0\right)$

$$
\Pi_{l} \dot{z}_{l}^{*}=\frac{z_{l}^{*}}{2\left(\lambda z_{l}^{*}+\mu\right)} \Pi_{l} \dot{\Pi}_{l}=\frac{z_{l}^{*}}{4\left(\lambda z_{l}^{*}+\mu\right)} \frac{\mathrm{d}}{\mathrm{d} t}\left(\Pi_{l}^{\mathrm{c}-}\right)^{2} \geqslant 0 .
$$

Thus, equation (56) can always be satisfied.

The critical condition for starting reorientation can be expressed by

$$
\Pi_{l}=\Pi_{l}^{\mathrm{c} \pm}
$$

where ' + ' denotes the forward progress $\left(\dot{\zeta}_{l}>0\right)$, and ' - ' the reverse progress $\left(\dot{\zeta}_{l}<0\right)$, and $\Pi_{l}^{\mathrm{c}+}>0, \Pi_{l}^{\mathrm{c}-}<0$.

Therefore the evolution equation for the reorientation process is

$$
\begin{array}{ll}
\dot{\Pi}_{l}=\dot{\Pi}_{l}^{\mathrm{c}+}=\lambda_{1} \dot{\zeta}_{l}, & \text { when } \dot{\zeta}_{l}>0 \\
\dot{\Pi}_{l}=\dot{\Pi}_{l}^{\mathrm{c}-}=\lambda_{1} \dot{\zeta}_{l}, & \text { when } \dot{\zeta}_{l}<0 .
\end{array}
$$

Here, the variation rate of the progress variable is also assumed to be proportional to the variation of the thermodynamic driving force. $\lambda_{1}$ is a material constant with a positive sign. If $\dot{\Pi}_{l}^{\mathrm{c}+}>0, \dot{\zeta}_{l}>0$; if $\dot{\Pi}_{l}^{\mathrm{c}-}<0, \dot{\zeta}_{l}<0$. Once the forward process starts, $\Pi_{l}^{\mathrm{c}-}$ moves back to its maximum value $\left(\Pi_{\mathrm{Re}}^{-}\right)$, where the subscript Re stands for reorientation. When the reverse process starts, $\Pi_{l}^{\mathrm{c}+}$ returns back to its minimum value $\left(\Pi_{\mathrm{Re}}^{+}\right)$. Here, $\Pi_{\mathrm{Re}}^{+}$and $\Pi_{\mathrm{Re}}^{-}$are material constants. Note that $\Pi_{\mathrm{Re}}^{+} \geqslant 0$ and $\Pi_{\mathrm{Re}}^{-} \leqslant 0$. In most cases, $\Pi_{\mathrm{Re}}^{+}=-\Pi_{\mathrm{Re}}^{-}=\Pi_{\mathrm{Re}}^{0}$. 
Referring to equation (63), in the forward process $\left(\dot{\zeta}_{l}>0\right)$

$$
\Pi_{l} \dot{\zeta}_{l}=\frac{1}{\lambda_{1}} \Pi_{l} \dot{\Pi}_{l}=\frac{1}{2 \lambda_{1}} \frac{\mathrm{d}}{\mathrm{d} t}\left(\Pi_{l}^{\mathrm{c}+}\right)^{2} \geqslant 0 ;
$$

in the reverse process $\left(\dot{\zeta}_{l}<0\right)$

$$
\Pi_{l} \dot{\zeta}_{l}=\frac{1}{\lambda_{1}} \Pi_{l} \dot{\Pi}_{l}=\frac{1}{2 \lambda_{1}} \frac{\mathrm{d}}{\mathrm{d} t}\left(\Pi_{l}^{\mathrm{c}-}\right)^{2} \geqslant 0 .
$$

Again, equation (56) is always satisfied.

Equations (29), (36), (38), (44), (57), (58), (62) and (63) are the basic governing equations for the phase transformation and reorientation in shape memory alloys.

\section{Conclusions}

A constitutive model for stress-induced phase transformation and reorientation in shape memory alloy single crystal is developed. This is the first time that both forward (reverse) phase transformation and reorientation are modeled under the same thermodynamic framework. Based on the principles of thermodynamics and the ensemble average method of micro-mechanics, the macroscopic Gibbs free energy is expressed as a function of the volume fractions of different martensite lattice correspondence variants (instead of habit plane variants); those variants are chosen as the internal variables to describe the microstructure configuration of shape memory alloys. Thermodynamic driving forces corresponding to the forward/reverse phase transformation and reorientation are derived from the macroscopic Gibbs free energy.

The total elastic strain energy includes two parts: the elastic strain energy caused by the applied external load in the absence of phase transformation eigenstrain, and the interaction energy induced by the internal stress. The internal stress comes from the phase transformation eigenstrain in the absence of external load. The second part is not directly dependent on the applied external load, but indirectly on the volume fractions of martensite lattice correspondence variants.

Mori-Tanaka average theory for randomly distributed two-phase mixture is extended to the $(n+1)$-component mixture to evaluate the interaction energy in the phase transformation. The interaction energy is expressed as a second-order polynomial function in terms of the volume fractions of the martensite lattice correspondence variants. The total number of independent material constants in the interaction energy is no more than the number of martensite lattice correspondence variants.

The critical condition and the evolution equation for the forward/reverse phase transformation and reorientation are obtained. It is proposed to describe the complex interior hysteresis loop by a critical thermodynamic driving force jump when the opposite transition takes place.

\section{References}

[1] Otsuka K and Wayman C M (ed) 1998 Shape Memory Materials (New York: Cambridge University Press)

[2] Otsuka K and Ren X 1999 Recent developments in the research of shape memory alloys Intermetallics 7511
[3] Kahn H, Huff M A and Heuer A H 1998 The TiNi shape-memory alloy and its applications for MEMS J. Micromech. Microeng. 8213

[4] Fu Y, Huang W, Du H, Huang X, Tan J and Gao X 2001 Characterization of NiTi shape-memory alloy thin films for MEMS applications J. Surf. Coat. Technol. 145107

[5] Fu Y Q, Du H J, Huang W M, Zhang S and Hu M 2004 TiNi-based thin films in MEMS applications: a review Sensors Actuators A 112395

[6] Turteltaub S and Suiker A S J 2006 A multiscale thermomechanical model for cubic to tetragonal martensitic phase transformations Int. J. Solids Struct. 434509

[7] Patoor E, Lagoudas D C, Entchev P B, Brinson L C and Gao X J 2006 Shape memory alloys, part I: general properties and modeling of single crystals Mech. Mater. 38391

[8] Lagoudas D C, Entchev P B, Popov P, Patoor E, Brinson L C and Gao X J 2006 Shape memory alloys, part II: modeling of polycrystals Mech. Mater. 38430

[9] Peultier B, Ben Zineb T and Patoor E 2006 Macroscopic constitutive law of shape memory alloy thermomechanical behaviour. Application to structure computation by FEM Mech. Mater. 38510

[10] Hall G J and Govindjee S 2002 Application of a partially relaxed shape memory free energy function to estimate the phase diagram and predict global microstructure evolution J. Mech. Phys. Solids 50501

[11] Niclaeys C, Ben Zineb T, Arbab-Chirani S and Patoor E 2002 Determination of the interaction energy in the martensitic state Int. J. Plast. 181619

[12] Govindjee S and Miehe C 2001 A multi-variant martensitic phase transformation model: formulation and numerical implementation Comput. Methods Appl. Mech. Eng. 191215

[13] Thamburaja P and Anand L 2001 Polycrystalline shape-memory materials: effect of crystallographic texture J. Mech. Phys. Solids 49709

[14] Siredey N, Patoor E, Berveiller M and Eberhardt A 1999 Constitutive equations for polycrystalline thermoelastic shape memory alloys. Part I. Intragranular interactions and behavior of the grain Int. J. Solids Struct. 364289

[15] Gall K and Sehitoglu H 1999 The role of texture in tension-compression asymmetry in polycrystalline NiTi Int. J. Plast. 1569

[16] Huang M and Brinson L C 1998 Multivariant model for single crystal shape memory alloy behavior J. Mech. Phys. Solids 461379

[17] Lu Z K and Weng G J 1997 Martensitic transformation and stress-strain relations of shape-memory alloys J. Mech. Phys. Solids 451905

[18] Boyd J G and Lagoudas D C 1996 A thermodynamical constitute model for shape memory materials. 1 . The monolithic shape memory alloy Int. J. Plast. 12805

[19] Lexcellent C, Goo B C, Sun Q P and Bernardini J 1996 Characterization, thermomechanical behaviour and micromechanical-based constitutive model of shape-memory $\mathrm{Cu}-\mathrm{Zn}-\mathrm{Al}$ single crystals Acta Mater. 443773

[20] Wechsler M S, Lieberman D S and Read T A 1953 On the theory of the formation of martensite Trans. Am. Inst. Min. Metall. Eng. 1971503

[21] Bowles J S and Mackenzie J K 1954 The crystallography of martensite transformations.1 Acta Metall. 2129

[22] Bowles J S and Mackenzie J K 1954 The crystallography of martensite transformations. 3. Face-centred cubic to body-centred tetragonal transformations Acta Metall. 2224

[23] Wayman C M 1964 Introduction to the Crystallography of Martensite Transformation (New York: MacMillan)

[24] Ball J M and James R D 1987 Fine phase mixtures as minimizers of energy Arch. Ration. Mech. Anal. 10013 
[25] Bhattacharya K 1992 Self-accommodation in martensite Arch. Ration. Mech. Anal. 120201

[26] Saburi T and Nenno S 1982 The shape memory effect and related phenomena Proc. Int. Conf. on Solid-Solid Phase Transformations ed H I Aaronson, D E Laughlin, R F Sekerka and C M Wayman, p 1455

[27] Zhu J J, Huang W M and Liew K M 2001 On the description of transformation in DO3 to 18R shape memory alloys using group theory IUTAM Symp. on Mechanics of Martensitic Phase Transformations in Solids ed Q P Sun (Dordrecht: Kluwer-Academic) p 163

[28] Shield T W 1995 Orientation dependence of the pseudoelastic behavior of single-crystals of $\mathrm{Cu}-\mathrm{Al}-\mathrm{Ni}$ in tension J. Mech. Phys. Solids $\mathbf{4 3} 869$

[29] Zhu J J and Liew K M 2004 Describing the morphology of 2H martensite using group theory part I: theory Mech. Adv. Mater. Struct. 11197

[30] Liew K M and Zhu J J 2004 Describing the morphology of 2H martensite using group theory part II: case study Mech. $A d v$. Mater. Struct. 11227

[31] Hane K F and Shield T W 1999 Microstructure in a copper-aluminium-nickel shape-memory alloy Proc. R. Soc. A 4553901

[32] Hane K F and Shield T W 1999 Microstructure in the cubic to monoclinic transition in titanium-nickel shape memory alloys Acta Mater. 472603

[33] Ericksen J L 1984 The Cauchy and Born hypotheses for crystals Phase Transformation and Material Instabilities in Solids ed M E Gurtin (New York: Academic) p 61

[34] Abeyaratne R, Chu C and James R D 1996 Kinetics of materials with wiggly energies: theory and application to the evolution of twinning microstructures in a CuAlNi shape memory alloy Phil. Mag. A 73457

[35] James R D and Hane K F 2000 Martensitic transformations and shape-memory materials Acta Mater. 48197

[36] Hall G J, Govindjee S, Sittner P and Novak V 2007 Simulation of cubic to monoclinic-II transformations in a single crystal CuAlNi tube Int. J. Plast. 23161

[37] Kruzik M, Mielke A and Roubicek T 2005 Modelling of microstructure and its evolution in shape-memory-alloy single-crystals, in particular in CuAlNi Meccanica 40389

[38] Govindjee S, Mielke A and Hall G J 2003 The free energy of mixing for $\mathrm{n}$-variant martensitic phase transformations using quasi-convex analysis (vol 50, p 1897, 2002) J. Mech. Phys. Solids $\mathbf{5 1} 763$

[39] Govindjee S, Mielke A and Hall G J 2002 The free energy of mixing for n-variant martensitic phase transformations using quasi-convex analysis J. Mech. Phys. Solids 501897

[40] Huang W 1999 'Yield' surfaces of shape memory alloys and their applications Acta Mater. 472769

[41] Smyshlyaev V P and Willis J R 1999 On the relaxation of a three-well energy Proc. R. Soc. A 455779
[42] Bhattacharya K 1993 Comparison of the geometrically nonlinear and linear theories of martensitic-transformation Contin. Mech. Thermodyn. 5205

[43] Zhu J J, Huang W M and Liew K M 2003 Deformation energy in martensitic transformation J. Physique IV 112179

[44] Hill R 1983 Interfacial operators in the mechanics of composite media J. Mech. Phys. Solids 31347

[45] Mori T and Tanaka K 1973 Average stress in matrix and average elastic energy of materials with misfitting inclusions Acta Metall. 21571

[46] Mura T 1982 Micromechanics of Defects in Solids (Dordrecht: Kluwer-Academic)

[47] Müller I and Xu H 1991 On the pseudo-elastic hysteresis Acta Metall. Et Mater. 39263

[48] Fedelich B and Zanzotto G 1991 One-dimensional quasi-static nonisothermal evolution of shape-memory material inside the hysteresis loop Contin. Mech. Thermodyn. 3251

[49] Fu S, Huo Y and Muller I 1993 Thermodynamics of Pseudoelasticity—an analytical approach Acta Mech. 991

[50] Huo Y and Muller I 1993 Nonequilibrium thermodynamics of pseudoelasticity Contin. Mech. Thermodyn. 5163

[51] Atanackovic T and Muller I 1995 A new form of the coherency energy in pseudoelasticity Meccanica $\mathbf{3 0} 467$

[52] Muller I and Seelecke S 2001 Thermodynamic aspects of shape memory alloys Math. Comput. Modelling 341307

[53] Seelecke S 1996 Equilibrium thermodynamics of pseudoelasticity and quasiplasticity Contin. Mech. Thermodyn. 8309

[54] Iadicola M A and Shaw J A 2002 The effect of uniaxial cyclic deformation on the evolution of phase transformation fronts in pseudoelastic NiTi wire J. Intell. Mater. Syst. Struct. 13143

[55] Shaw J A 2002 A thermomechanical model for a 1D shape memory alloy wire with propagating instabilities Int. J. Solids Struct. 391275

[56] Hill R 1972 Constitutive macro-variables for heterogeneous solids at finite strain Proc. R. Soc. A 326131

[57] Willis J R 1991 Mechanics of Composites, private communication, University of Cambridge

[58] Hill R 1963 Elastic properties of reinforced solids—some theoretical principles J. Mech. Phys. Solids 11357

[59] Willis J R 1983 The overall elastic response of composite-materials J. Appl. Mech. Trans. ASME 501202

[60] Gao X J, Huang M S and Brinson L C 2000 A multivariant micromechanical model for SMAs Part 1. Crystallographic issues for single crystal model Int. J. Plast. 161345

[61] Zhu J J 1998 Constitutive theory of martensite phase transformation in shape memory alloy Postdoctoral Research Report (Beijing: Institute of Mechanics, Chinese Academy of Sciences) 